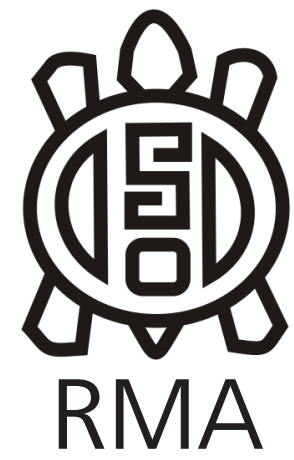

Dossier

\title{
Privación de libertad no significa privación de identidad. Experiencias educativas en espacios carcelarios. Museo Histórico Nacional, Chile
}

\author{
Deprivation of liberty does not mean deprivation of identity. Educational \\ experiences in prison spaces. Museo Histórico Nacional, Chile
}

Leonardo Mellado González*, Mauricio Soldavino Rojas**, Pablo Soto Gonzalez***, Marcela Torres Hidalgo****

\begin{abstract}
*Jefe del área de Educación y Mediación, Museo Histórico Nacional./Coordinador Programa de Intervención Patrimonial (PROIP), Facultad de Ciencias Sociales, Escuela de Historia, Universidad

Academia de Humanismo Cristiano, Santiago de Chile. E-mail: leonardo.mellado@mhn.gob.cl-leondynis@yahoo.es **Unidad de Educación, Área de Educación y Mediación, Museo Histórico Nacional. E-mail:mauricio.soldavino@mhn.gob.cl ***Unidad de Educación, Área de Educación y Mediación, Museo Histórico Nacional. E-mail:pablo.soto@mhn.gob.cl ****Coordinadora Unidad de Educación, área de Educación y Mediación, Museo Histórico Nacional. E-mail:marcela.torres@mhn.cl
\end{abstract}

\begin{abstract}
Resumen
Muchos son los servicios que los museos pueden ofrecer a sus visitantes, sin embargo, cuando la accesibilidad a estos lugares se ve restringida a aquellas personas que, por distintas razones, se encuentran privadas de libertad en recintos penitenciarios, la acción de estas instituciones culturales queda también limitada por diversas razones como el desconocimiento o simplemente el desinterés de responder a un derecho básico de todas las personas como es el de la identidad cultural por medio del acceso al patrimonio. Es por ello que la Unidad Educativa del Museo Histórico Nacional, busca promover el respeto a la diversidad cultural de nuestro país y entregar a sus comunidades, sin exclusión de alguna de ellas: étnica, social, religioso etc., conocimiento de la historia de Chile y del patrimonio que custodia a través de la actividad "Los objetos de mi historia" que forma parte del programa: "El Museo sale del Museo".
\end{abstract}

Palabras claves: Museo; Privación de la libertad; Patrimonio; Educación.

\begin{abstract}
Many are the services that the museums can offer to its visitors, however, when the accessibility to this places it's restricted to those people who for different reasons are confined in different places as, children's home, nursing home and especially the penitentiary, the actions of this cultural institutions is limited, not being able to response to a basic right, the cultural identity by the access to the cultural heritage. It is therefore that the Education Department of the National Historic Museum, looks to promote the respect to the cultural diversity of our country and give to the communities, without exclusion of some of them: Ethnic, social, religious, etc., knowledge of the history of Chile and the custody of the cultural heritage in attention of its mandateby the activity "The objects of my history" which is part of the program "The Museum outside the Museum".
\end{abstract}

Keywords: Museum; Deprivation of liberty; Heritage; Education.

\section{Introducción}

Como Unidad de Educación del $\mathrm{MHN}^{1}$, es fundamental aportar al proceso de reinserción de las personas en

\footnotetext{
1 Museo Histórico Nacional, MHN en adelante.
}

situación de encierro desde un ámbito que involucre sus memorias y la valoración de éstos como sujetos históricos, lo que en muchos casos los ayuda a seguir adelante con la esperanza de que puedan insertarse nuevamente en una sociedad que los ha excluido permanentemente por su actuar, pero que no ha hecho lo suficiente para darles un 
futuro diferente al que les ha tocado vivir desde su niñez. Y como señalamos en el presente título, la privación de libertad no significa necesariamente privación de la propia identidad cultural expresada a través del Patrimonio Cultural y entendida como "un componente esencial entre los seres humanos y su entorno, tanto natural como cultural, posible de identificar como tal y de ser inscrito a manera de proceso al interior de una narrativa histórica" (Andrade, 2018, p.111), o como señala el sociólogo J. Larraín, hablar de identidades es referirse a "un proceso de construcción en la que los individuos se van definiendo a sí mismos en estrecha interacción simbólica con otras personas" (Larraín, 2003, p. 32). Pero si el patrimonio (material e inmaterial) que resguarda y difunde el museo, así como sus prácticas, permanecen encerradas dentro de sus paredes, como ocurre gran parte de los museos, esa relación se vuelve difícil, sino imposible.

Es por ello que los museos deben, no solo acoger el llamado formulado por la Nueva Museología per se -entendiendo esto como el «Diálogo entre sujetos», basado en la participación activa de los miembros de la comunidad, en donde el museólogo deja de contemplarse como el experto encargado de dirimir la verdad, para convertirse en un «catalizador» al servicio de las necesidades de la comunidad (Alonso, 2001)-, sino también al de sus propios ciudadanos, quienes demandan mayor participación en su relación con las instituciones culturales como los museos.

\section{Sobre la Institución}

El MHN, ${ }^{2}$ fue creado por mandato del presidente de la República, Ramón Barros Luco, el 2 de mayo de 1911. Es uno de los tres museos nacionales, siendo parte, desde su creación, del Ministerio de Educación y desde marzo de 2018, del recientemente creado Ministerio de las Culturas, las Artes y el Patrimonio.

La misión institucional del Museo Histórico Nacional es "facilitar a la comunidad nacional e internacional el acceso a la comprensión de la historia del país, permitiendo el reconocimiento de las diversas identidades que lo

\footnotetext{
2 Dentro de su mandato fundante en el Artículo 21.- del DFL Núm. 5,200.- de Santiago, 18 de Noviembre de 1929, "El Museo Histórico Nacional reunirá todos los objetos relacionados con la historia patria, tanto civil como militar, y con el ambiente y las costumbres de Chile en sus diversas épocas. En su sección de prehistoria, se limitará al aborigen chileno, con lo cual, en conformidad al artículo anterior, constituirá la colección de base y preferencia en el ramo, dentro del país." Definiendo con ello su acerbo patrimonial.

A su vez, dicho decreto en su sección 6.o, referido a las diversas instituciones patrimoniales que pertenecían a la Dibam, entre las que se encuentra el Museo en cuestión señala: "- Que dichos establecimientos, aun cuando no sean docentes, pueden y deben cooperar con eficacia a la enseñanza nacional, divulgando por todos los medios a su alcance los tesoros de sus colecciones y los resultados de sus investigaciones y estudios, como asimismo procurando a los establecimientos educacionales elementos que les pueden elaborar y proporcionar;" (Ley 21045 Art. 57 N 1 D.O. 03.11.2017, con base en el DFL Núm. 5,200.- de Santiago, 18 de Noviembre de 1929).
}

constituyen y dan forma a Chile, desde su pasado precolombino a su conformación política y territorial". Por lo que, haciendo eco de su mandato inicial, el facilitar a toda la comunidad el acceso a la comprensión de la historia chilena, no es más que un reforzamiento de su propósito, el cual creemos se cumple cuando hablamos y trabajamos con personas de las escuelas penitenciarias.

Debemos reconocer eso sí, que el equipo de profesionales que han desempeñado funciones en el área de educación del museo, decidieron dar respuesta a una necesidad educativa y personal de buscar nuevos públicos sin discriminar para responder ante el mandato institucional y al personal, como profesionales de la educación patrimonial.

Por su parte, la Unidad Educativa del MHN, busca promover el respeto a la diversidad cultural de nuestro país y entregar a las diversas comunidades, sin exclusión de alguna de ellas: étnica, social, religiosa, etc., conocimiento de la historia de Chile y del patrimonio. Además, pretende que nuestro público diverso comprenda, valore y se sienta partícipe como sujeto histórico en la construcción de identidad y memoria del país. Procura contribuir al desarrollo de conocimientos y habilidades para que todos/ as puedan interpretar objetos patrimoniales a partir de su condición de fuente histórica. Finalmente contribuir a que nuestros usuarios comprendan el carácter social y dinámico del patrimonio cultural.

Es así como, hacia el año 2000, los educadores del MHN se preguntaron: ¿Existe una relación equitativa o igualitaria entre el Patrimonio resguardado y difundido por el MHN y su(s) comunidad(es)? Impulsados, por tanto, por un deseo de responder a esta pregunta es que en adelante se desarrollaron diversas iniciativas que apuntaban a acercar a diversos grupos de públicos, atendiendo sus necesidades y las de la institución.

Entre estas actividades se cuentan: capacitaciones a docentes; atención a escuelas de ciegos y sordos; desarrollo de material educativo; desarrollo de guion alternativo al de la muestra permanente con "enfoque de género", el que además fue parte de una exigencia pública como meta institucional por medio de los llamados Proyectos de Mejoramiento a la Gestión (PMG); trabajo con comunidades locales realizado entre los años 2005-2008 con pobladores de La Legua ${ }^{3}$, entre otras, las que se sumaban a las visitas guiadas mediadas, a través de diversos ejercicios con objetos patrimoniales en una sala acondicionada especialmente para este propósito, llamada Sala didáctica, creada en la década de los 80.

\footnotetext{
3 La población La Legua, es una comunidad de vecinos o barrio, ubicado al centro-sur de Santiago, constituida a partir de tomas de terrenos desde finales de 1920, altamente estigmatizada, pero con una fuerte identidad local, con la que se desarrollaron diversas actividades tanto en su territorio como dentro del museo, con cuyos vecinos se desarrollaron diversas actividades como exposiciones, temporales, seminarios y talleres de memoria.
} 
Nuestra convicción es que el museo como institución pública y nacional, se debe enfocar no solamente en los usuarios que visitan el edificio Palacio de la Real Audiencia -edificación colonial de 1808 que alberga el museo-, sino que también es imprescindible salir a realizar actividades patrimoniales a comunidades que no han tenido la posibilidad de visitar el museo por diversos motivos.

Es así que hacia el 2010 y como consecuencia de una singular coyuntura como el Terremoto que afectó gran parte de las zonas del centro sur de Chile $\left(27 \mathrm{~F}^{4}\right)$, pero con la claridad de que este era un paso a desarrollar, el equipo de educación del Museo Histórico Nacional de Chile generó una interrogante que clamaba por ser contestada: ¿Es este el momento de realizar actividades educativas patrimoniales a estudiantes y personas en general en contextos de encierro o privación de libertad?

La respuesta no se dejó esperar, ¡Sí, a todos los públicos sin exclusión! Por lo que, las metas de atención de público, y por sobre todo, mantener viva la relación con la comunidad, naciendo así el programa "El Museo sale del Museo", permitiendo con ello desarrollar, de manera permanente, actividades hacia: escolares vulnerables; tercera edad; escuelas hospitalarias; migrantes; personas pertenecientes a Corporaciones de Salud Mental COSAM; estudiantes con retos múltiples; personas con visión reducida y total; niños y adultos hospitalizados con problemas neurológicos y enfermedades psiquiátricas; jóvenes condenados pertenecientes al Servicio Nacional de Menores SENAME y desde el año 2012 la actividad: Escuelas penitenciarias, inclusión a través de la historia, museos y patrimonio, pensada en estudiantes adultos privados/as de libertad, quienes entre sus derechos internacionales está: "Participar en actividades culturales y educativas encaminadas a desarrollar plenamente la personalidad humana". (Naciones Unidas, 1990)

Es aquí donde nuestra misión como institución pública se debe conectar con otras instituciones del mismo Estado, en este caso Gendarmería de Chile ${ }^{5}$, para fortalecer las políticas que busquen generar cambios positivos y valoración de su pasado a este tipo de población que se encuentra en las cárceles y que el mismo Estado ha fallado en la aplicación de políticas públicas que ayuden a disminuir la brecha entre ricos y pobres, porque finalmente un preso que está estudiando significa que en su niñez o juventud no tuvo las mismas oportunidades que alguien que logró terminar sus estudios básicos y secundarios, por lo que se debe potenciar sus habilidades, capacidades

\footnotetext{
${ }^{4}$ Como se le llama comunicacionalmente.

${ }^{5}$ Gendarmería de Chile es un Servicio Público dependiente del Ministerio de Justicia y Derechos Humanos, que "tiene por finalidad atender, vigilar y contribuir a la reinserción social de las personas que por resolución de autoridades competentes, fueren detenidas o privadas de libertad y cumplir las demás funciones que les señala la ley" Artículo 1 de la Ley Orgánica de Gendarmería de Chile.http:// www.gendarmeria.gob.cl/
}

para salir adelante, ya que al momento de que obtengan su libertad, tendrán más herramientas para desenvolverse dentro de una sociedad que no brinda mucho apoyo a las personas que han tenido un pasado delictual.

Desde un comienzo entendimos que la privación de libertad no significa necesariamente privación de la propia identidad cultural, expresada o representada a través del Patrimonio Cultural. Pero, si ese patrimonio permanece encerrado dentro las paredes de un museo, esa relación se vuelve imposible.

A su vez, no podemos perder de vista que el acceso al Patrimonio Cultural es un derecho establecido en la propia Constitución política de la nación en donde se señala en su articulado que:

\section{"Artículo 19 de la CPCH}

$10^{\circ}$.- El derecho a la educación.

"La educación básica y la educación media son obligatorias, debiendo el Estado financiar un sistema gratuito con tal objeto, destinado a asegurar el acceso a ellas de toda la población. En el caso de la educación media este sistema, en conformidad a la ley, se extenderá hasta cumplir los 21 años de edad.

Corresponderá al Estado, asimismo, fomentar el desarrollo de la educación en todos sus niveles; estimular la investigación científica y tecnológica, la creación artística y la protección e incremento del patrimonio cultural de la Nación."

Con esto, más el deber institucional que pesa sobre el museo, no hay excusas para proseguir con el trabajo en las escuelas y liceos penitenciarios.

\section{Realidad carcelaria en Chile}

Hasta agosto de 2016 la población penal era de 107.261 personas, de las cuales 49.063 (45.7\%) están en régimen cerrado (Bulnes, F., et al, 2017). Los internos tienen pena privativa de libertad debido a: condenas por hurto, por un valor menor a $\$ 185.000$ (247,90 EUR); conductores en estado de ebriedad, traficante menor de drogas, etc.

La población penal condenada fundamentalmente corresponde a delitos contra la propiedad con 51\%, por delitos contra la vida (8\%) y o integridad física de las personas 7\%. (Bulnes et al. 2017).

Las características de los/as chilenos/as privados de libertad corresponden a "las características sociales y demográficas que dan cuenta de un grupo relativamente homogéneo en términos de educación y empleo, con indicadores de una educación bajo la media del país" (Bulnes et al. 2017, Collarte et al 2017), Solo el 14\% completó la educación media y $7.3 \%$ es analfabeta. Se expresa en las condiciones de exclusión social, carentes 
de educación, salud, oportunidades, trabajo etc.

La población penal es mayoritariamente masculina sobre el $90 \%$, siendo el promedio los 33 años.

En el año 2016 funcionaban 89 establecimientos educativos penitenciarios de enseñanza básica y media a lo largo del país. El 70\% imparte enseñanza básica y media; el 30\% restante solo enseñanza básica. Los objetivos que llevan al interno a postular a las escuelas penitenciarias se deben a diversas causalidades, ejemplo, méritos para beneficios carcelarios como salida dominical, continuación de estudios, etc.

Para el año 2015 los hombres que cursaron enseñanza básica (primaria) fueron 6.159, mientras que las mujeres fueron 646, dando como resultado un total de 6.808 estudiantes. Los internos que asistieron al liceo, cursando enseñanza media (secundaria) fueron: hombres 8.206; mujeres 701 siendo el total de 8.907. Sumando un total de estudiantes de escuelas penitenciarias de varones; 14.365 y mujeres 1.350 , con un total de 15.715 estudiantes. 176 son estudiantes en Educación Superior (Garcés, H., De Rosas, N., et al, 2016).

\section{El trabajo del área educativa en los centro de educación penitenciaria}

Así como el resto de las actividades, programas y experiencias desarrolladas por la Unidad, el trabajo de educación con las escuelas penitenciarias reciben el nombre de "Los objetos de mi historia" del programa "El Museo sale del museo".

Para crear la actividad para estudiantes de liceos penitenciarios se realiza una revisión sobre actividades museales en liceos penitenciarios en Chile y en el extranjero y entrevistas a encargados del área de educación de Gendarmería de Chile; y además, se recurrió a bibliografía relacionada a temas de educación carcelaria en textos como el de Bulnes, F., Collarte, C., Fruhling, H., Mardones, C., Ramm, A., Roblero, L., Santibáñez, Sanhueza, G., Singer., M., Tello, C., \& Vial, P.(2017). Sistema carcelario en Chile: propuestas para avanzar hacia una mayor efectividad y re inserción.del Centro de Políticas Públicas de la UC. Y del texto del Ministerio de Educación para adultos en encierro de Garcés, $H_{\text {., }}$ De Rosas, N., Gacitúa, D., Pedraza, M., \& Infante, I. (04,2016). Educación para la libertad. Propuesta de mejoramiento y pertinencia de los procesos pedagógicos para establecimientos educacionales en contextos de encierro, 2017, de la Coordinación Nacional de Educación de Personas Jóvenes y Adultas, correspondiente a la División de Educación General.

\section{a) Acciones realizadas para diseñar la experiencia}

Junto a entrevistas semiestructuradas a docentes de los liceos penitenciarios, evaluaciones realizadas también a los docentes de los liceos, bitácora realizada a las actividades realizadas en los liceos por docentes del MHN. Junto a esto, también en forma paralela se llevó a cabo un estudio de las metodologías educativas por un equipo externo al museo en el año 2014 (Marsal,D., y equipo, 2014).

Los recintos de la región metropolitana visitados han sido seis, desde el año 2012 al 2017 inclusive, siendo el programa ejecutado 13 veces (Santiago, Ex penitenciaría Liceo Herbert Vargas Wallis (hombres), en 4 ocasiones; Puente Alto, Liceo Camino de Luz (hombres), visitada 2 ocasiones; San Miguel, Escuela Cárcel Hugo Morales Bizama (mujeres), visitado en tres ocasiones; Colina 1, Liceo Rebeca Olivares, visitado dos veces (hombres); Talagante, Liceo Presidente Prieto (hombres) y San Joaquín Liceo Santa María Eufrasia (mujeres) ambos visitados una vez. En total, 891 estudiantes internos han participado en la actividad.

\section{b) Antecedentes en el sistema educativo}

Los establecimientos educacionales de encierro son diferentes entre ellos, a pesar que tienen en común la dependencia tripartita: Gendarmería de Chile (que hace de apoderado), las corporaciones municipales ${ }^{6}$ a las que pertenece cada establecimiento educacional y el Ministerio de Educación (Mineduc) con su propuesta ministerial Educación para la Libertad, con la que busca mejorar la calidad de los procesos pedagógicos para establecimientos educacionales en contextos de encierro, quien señala que:

“...se debe favorecer un ambiente grato para el aprendizaje, es decir, a pesar de las dificultades, desarrollar la confianza en la capacidad y el deseo de aprender en los estudiantes, en su creatividad y autonomía frente al aprendizaje; (...) promover situaciones educativas que desafíen la capacidad de aprender en los estudiantes y la reflexión acerca de los resultados de sus propios aprendizajes, y lograr la colaboración de otras instituciones para el logro de sus fines educativos. Los internos tienen derecho a una educación pertinente y de calidad". (Garcés, H., De Rosas, N., 2016)

\section{c) Lo que motiva la propuesta}

El fracaso de las políticas carcelarias para alcanzar una real reinserción social de las personas que se encuentran privadas de libertad, nos motivó a trabajar con estudiantes en contexto de encierro, porque consideramos que son un grupo que ha sufrido por la falta de oportunidades, permanentemente excluidos por la sociedad, viven en los ghettos periféricos, muchos creados por el propio Estado, donde la delincuencia y la droga son parte de sus vidas y

\footnotetext{
${ }^{6}$ Municipalidades de: Santiago, Puente Alto, San Joaquín, San Miguel, Colina y Talagante.
} 
el no validarse bajo este designio les significa convertirse en el extraño de la población. Sin embargo, creemos como Unidad de Educación, que es nuestra responsabilidad ir a brindar actividades de calidad a los jóvenes y adultos en condición de encierro, escucharlos y acogerlos con nuestro programa. Como profesionales de la educación aceptamos el desafío de trabajar con nuevas audiencias y en particular con aquellas que terminan siendo más excluidas.

En el actual contexto de inclusión a personas con capacidades diferentes, minorías culturales, sexuales, entre otras, implica realizar una autocrítica para quienes trabajamos en museos. Muchas veces, porque solo nos dedicamos a esperar la llegada de los usuarios, otras veces por temor e incluso por ignorancia, hemos mantenido marginados de los servicios culturales de nuestras instituciones a las personas privadas de libertad. En el año 2012, el Museo Histórico Nacional se convirtió en el primer museo de Chile en aceptar este desafío e incluir esta audiencia considerándolos beneficiarios de los servicios educativos culturales del museo. Todo ello nos convierte en los pioneros en realizar actividades patrimoniales en forma permanente en las escuelas penitenciarias de Chile?

En Europa (Vidal, T\&H Ruiz, M. 2014) hay presencia de museos de artes e historia natural realizando actividades con personas privadas de libertad, pero no observamos museos históricos. En nuestro país en este momento somos los únicos que tenemos un programa de inclusión en contexto de encierro, en el que trabajamos: los oficios, memoria, historia y patrimonio. Realizamos además un trabajo colaborativo a la educación formal, favoreciendo experiencias de aprendizaje significativo, aportando en la recuperación del sentido positivo de la vida, de la confianza en sí mismo, goce, identificación con el patrimonio nacional e incentivar el conocimiento de los oficios y herramientas como elemento identitario y de memoria de cada uno de ellos/as, lo que finalmente promueve la inclusión de estas personas que necesitan reinsertarse en la sociedad que los ha relegado al encierro.

\section{d) Fundamentos específicos de la propuesta}

Consideramos importante la reflexión, el aprendizaje significativo, los aportes personales que enriquecen el aprendizaje de cada uno, sus emociones y su pasado familiar, sus historias de vida que representan a un sector social postergado a lo largo de los años, pero también un sector que es parte relevante de la historia, por lo que es trascendental el trabajo de la memoria colectiva que representa a estos sectores que no son parte de la llamada "historia oficial" 8 , sino que son parte de

\footnotetext{
${ }^{7}$ La presente actividad ha sido un trabajo sistemático y sostenido en el tiempo a diferencia de experiencias similares en otros museos de Chile.

8 Entendemos por "historia oficial" como aquella que construye y narra el pasado con el objeto dejustificar históricamente el oficialismo,
}

esa historia desde abajo. "La historia es memoria y sin memoria no hay historia" (Garcés, 2001), ratificando que la memoria de las personas, es fundamental para que los internos puedan valorar su propia historia a través de objetos que los representan, así como entender que los aspectos emocionales de las experiencias educativas también pueden ser significativos.

\section{e) Fundamentos de la Metodología}

Nuestro taller se enriquece con la utilización de una metodología de trabajo basada en la teoría constructivista, la cual postula que son los estudiantes los que construyen su propio conocimiento a partir del contraste de las nuevas vivencias con las que ellos ya poseen. Como también pone énfasis en el reconocimiento del valor de los objetos que el museo resguarda como los objetos que se llevan a la actividad, los que están pensados para que ellos evoquen recuerdos personales y/o familiares, como fuente histórica que puede ser interpretada, metodología denominada como la "didáctica del objeto".

\section{f) Descripción de la actividad}

La actividad se realiza en las salas de clases de cada grupo de internos y cuenta con cuatro tiempos para su ejecución en el lapso de hora y treinta minutos:

1) Presentación y bienvenida a todos los participantes de forma empática y con respeto, presentamos a nuestra institución.

2) Museos e Interpretación histórica de un objeto. El objetivo de este momento es acercar a la audiencia al tema de la interpretación patrimonial a través de los objetos. Explicamos la importancia del Museo Histórico Nacional como institución de resguardo del patrimonio histórico y cultural de nuestro país.

La actividad se inicia con la siguiente pregunta: ¿Conocen o han visitado alguna vez algún Museo?, ¿Dónde está la Plaza de Armas de Santiago?, ¿Qué funciones piensan que tiene el MHN? A partir de las respuestas entregadas por los estudiantes abordamos el concepto: objeto como fuente histórica. Les contamos que los objetos que colecciona el museo son fuentes históricas y nos permiten recrear la historia de Chile y conocer nuestra cultura, es por eso que se colecciona, cuida, educa, exhibe y entretiene.

Es en este momento que les expresamos que la historia la escribimos todos y todas, que ellos también son sujetos históricos y lo que puedan aportar a la sociedad va a constituir una huella en nuestra cultura porque todos hacemos historia. Luego, a partir de una historia ficticia,

\footnotetext{
el Estado o la clase gobernante, muchas veces consensuada y claramente sesgada en cuanto a énfasis, procesos, temporalidades y sujetos históricos.
} 
les mostramos un cofre donde escondemos un objeto valioso, un tesoro de nuestra familia (de los profesores) el cual ha pasado de generación en generación -Esto lo hacemos para que comprendan que la historia no se limita solamente a objetos de valor comercial y personajes conocidos-. Procuramos que el relato incluya las características que más tarde les van a permitir evidenciar su valor histórico y patrimonial. Luego de todo lo anterior les preguntamos: ¿Ustedes creen que el objeto que hemos presentado podría estar en el museo?

A partir de sus respuestas abordaremos algunas características que permiten interpretar su historia, "un grupo de personas se siente reflejada y representadas en él", "nos entrega algunas pistas y datos históricos" y "nos entregan valores y de características de personas que vivieron antes que nosotros". Con cada una de las afirmaciones se solicita a alumnos participar sacando de algunos objetos las pistas (tarjetas) que deberán relacionar con la historia ficticia. Y para confirmar el aprendizaje, solicitamos cuatro voluntarios para realizar un ejercicio de análisis de objetos donde deberán organizar de más antigua a más moderna un grupo de objetos de una misma familia.

3) Trabajo grupal: Actividad dirigida por un profesor del MHN, momento en el que se busca poner en práctica lo aprendido anteriormente y para ello se trabaja con algunos objetos e imágenes de nuestra colección, los que se encuentran en cada baúl y que fueron pensados para ellos, quienes los conocen, incluso algunos trabajan o trabajaron alguna vez esos oficios. Es así que en estas reflexiones que surgen de forma espontánea sus recuerdos, sus memorias de infancia, esta instancia resulta enriquecedora ya que aportan con saberes traídos desde sus ancestros. Historias que los transportan a su época de niños.

El curso se divide en tres equipos de trabajo, cada uno de los cuales investigará un baúl. Pretendemos obtener de los estudiantes uno de los siguientes conceptos: oficios, herramientas y tradición; vida cotidiana; la carpintería, la nobleza de la madera. Cada grupo de trabajo abordará la temática a partir del relato del profesor, el que será apoyado con objetos alusivos a cada tema. La idea es aplicar las técnicas de interpretación histórica de los objetos antes vistos: ¿cómo se llama el objeto? ¿Paraqué sirve? ¿De qué material está hecho? ¿Se usa actualmente? ¿Qué recuerdos tengo de ellos? Etc.

Al finalizar, cada grupo debe elegir a representantes o todos ellos, los que exponen al resto del curso lo que aprendieron y concluyeron en su equipo. Para su explicación utilizan los objetos e imágenes que trabajaron.

4) Cierre de la actividad y evaluación formativa: Para finalizar, invitamos a los/as alumnos/as a realizar un plenario donde se avaluará la actividad, respecto a si creen que les sirvió aprender sobre los objetos patrimoniales como fuente histórica; los oficios como elemento identitario de una cultura; las herramientas y el trabajo artesanal; y el museo como institución de resguardo del patrimonio cultural de Chile y el trabajo de memoria personal y familiar.

Para terminar, los invitamos a tocar, observar e interpretar los objetos históricos expuestos en la mesa central. Nos despedimos y les agradecemos su buena disposición para participar en la actividad junto con invitarlos para que cuando puedan ellos y sus familias visiten el museo.

\section{Conclusiones}

Por medio de estas acciones creemos que como Unidad encargada de vincularnos con nuestras comunidades, damos un paso fundamental en dar accesibilidad a las personas en condición de encierro, privadas de libertad en recintos penitenciarios, respondiendo con ello a un derecho que consideramos fundamental como es el de la identidad cultural por medio del acceso al patrimonio.

Asimismo, estamos ciertos que las metodologías de acción aplicadas con las personas privadas de libertad, por medio de algunas actividades ejecutadas por el Departamento Educativo del Museo Histórico Nacional, han servido de ejemplo del trabajo desarrollado en contextos carcelarios, también replicados por otras instituciones museales, quienes han decidido incorporar dentro de sus prácticas a sujetos y metodologías divulgadas por nuestra unidad. Entre estas instituciones destacan el Museo de la Educación Gabriela Mistral, el Museo Violeta Parra, Museo de Artes Decorativas, Museo Nacional de Bellas Artes, el Museo Nacional de Historia Natural y el Museo de Historia Natural de Valparaíso.

En cuanto a los participantes y según nuestra experiencia en las distintas escuelas-liceos penitenciarios, los o las estudiantes agradecen y se motivan con visitas de personas externas al lugar de reclusión porque es una forma para interactuar con otras personas distintas al encierro como gendarmes y profesores de sus liceos y de esa manera, salir, aunque sea un rato, del contexto carcelario del que están envueltos en el día a día. Por ejemplo, en una de las visitas a la cárcel de Colina I, uno de los internos comentaba que "estas actividades son una motivación especial, ya que aprendemos algo nuevo, nos sentimos valorados como personas y además es una instancia para que podamos hablar de otros temas ajenos al contexto de encierro." Este mismo interno dijo "que la rutina diaria y mis conversaciones no salen del tema delictual, temas de la población, o de otras cárceles", por lo que la presencia de actividades educativas de personas e instituciones externas es algo que valoran y los motiva a participar con gran entusiasmo, incluso como algo nuevo que pueden contar a sus familiares los días de visita. 
Otros ejemplos de la actividad que realizamos es valorada por parte de los internos, son cosas que ellos mismos nos han contado, como acordarse de que vinieron al Museo cuando eran jóvenes o incluso el caso de una ex interna que asistió junto a su hijo el 29 de mayo 2017 al Día del Patrimonio Cultural, comentando que la había marcado de buena manera la actividad en la que participó cuando era estudiante de una escuela penitenciaria y que la motivó a interesarse más en profundidad de la temática relacionada con el patrimonio.

Además, los estudiantes valoran el trato que se les brinda en estas actividades, siempre de manera cordial, de igual a igual, lo que se manifiesta en un gran respeto por parte de ellos, desde los más jóvenes hasta los de mayor edad. El respeto y la confianza en ellos es una manera de demostrar que, a pesar de haber cometido errores en sus vidas, siempre hay una segunda oportunidad, y que la violencia con la que muchos de ellos conviven o han convivido en sus entornos y poblaciones, no solo es producida por ellos, sino que más bien sus condiciones de vida precarias, los han llevado a comportarse de manera violenta. Por tal motivo, es que el respeto es algo que ellos valoran y lo demuestran en las distintas actividades que hemos realizado, ya que no nos corresponde juzgarlos por sus errores, sino que los respetamos y queremos influir en su reinserción, brindándoles herramientas para que cuando obtengan su libertad, puedan seguir un mejor camino.

Otro aspecto importante es el reconocimiento a la memoria de todas las personas con las que trabajamos ya que muchos de los objetos que usamos hablan de las historias de los oficios, de los trabajadores, mineros, del campo, vida cotidiana, genero, de nuestros pueblos originarios etc., con lo que muchos se sienten identificados, haciéndolos recordar su pasado y la de sus familias, por lo que la memoria es una muy buena herramienta para que valoren su pasado como algo importante y que les brinda una identidad dentro de un contexto de encierro.

Al reconocer la memoria de los estudiantes de las Escuelas Penitenciarias también estamos aprendiendo de ellos, de sus historias, de sus formas de vida, de las injusticias y desigualdades con las que han crecido y que los diferentes gobiernos de turno y la clase política en general, no se han hecho cargo en la prevención del delito, en temas educacionales y sociales, para así poder brindarles un futuro con herramientas para que puedan surgir y salir de su condición de pobreza. Esto último influye de sobremanera en que muchos busquen como salida de su vulnerabilidad, el cometer delitos y con esto se inicia un círculo vicioso que no terminará si no se interviene desde las bases sociales de la que está compuesta la sociedad actual.

Para trabajadores de museos que en el futuro deseen desarrollar actividades con sus instituciones en recintos penitenciarios, se les recomienda como primer punto derribar los prejuicios sobre las personas con las que desarrollarán sus actividades. Para ello se debe desarrollar un acercamiento previo de entrevistas a carceleros y reos a fin de entender a los segundos primero como personas y no como delincuentes. Como sociedad debemos hacernos cargo de la reinserción de los presos, donde no basta solo una buena conducta, o palabras de aliento. Es preciso también enseñar que somos todos parte de una sociedad y eso precisamente es lo que puede lograrse a través del derecho a la identidad y al patrimonio.

Santiago de Chile, 10 de Junio 2018

\section{Blibliografía}

Alonso Fernández, Luis (2001). Museología y museografía. Barcelona: Ediciones del Serbal.

Andrade, Pablo; Mellado, Leonardo; Rueda, Hugo; Villar, Gabriela (2018) El Museo Mestizo, Fundamentación Museológica y Disciplinar para el Cambio de Guion. Museo Histórico Nacional.

Bulnes, F., Collarte, C., Fruhling, H., Mardones, C., Ramm, A., Roblero, L., Santibáñez, Sanhueza, G., Singer., M., Tello, C., \& Vial, P. (2017). Sistema carcelario en Chile: propuestas para avanzar hacia una mayor efectividad y re inserción. 2017, de Centro de Políticas Públicas UC Sitio web: http://politicaspublicas.uc.cl/wp-content/ uploads/2017/05/Art\%C3\%ADculo-Sistema-carcelarioen-Chile.pdf

Constitución Política de la República de Chile (1980). Texto actualizado a mayo de 2017. HCD.

Decreto con fuerza de ley $N^{\circ}$ 5.200, de 1929, del Ministerio de Educación Pública. https://www.leychile. $\mathrm{cl} /$ Navegar?idNorma=129136

Garcés, H., De Rosas, N., Gacitúa, D., Pedraza, M., \& Infante, I. (04,2016). Educación para la libertad. Propuesta de mejoramiento y pertinencia de los procesos pedagógicos para establecimientos educacionales en contextos de encierro. Junio 20, 2017, de Coordinación Nacional de Educación de Personas Jóvenes y Adultas. División de Educación General. Sitio web: http://epja.mineduc.cl/wpcontent/uploads/sites/43/2016/07/Educaci\%C3\%B3npara-la-libertad-2016-2018-Final-042016.pdf

Garcés, Mario (2001) "Memorias de la Dictadura en La Legua". ECO Educación y Comunicaciones, Santiago.

Larraín, Jorge (2003). El concepto de identidad. En Revista Famecos núm. 21.

Marsal, D., \& Equipo, (2014). Estudios de metodologías 
aplicadas por el área educativa Museo Histórico Nacional. Licitación 49-17LE14.

Naciones Unidas, Asamblea General "Principios básicos para el tratamiento de los reclusos", principio $N^{\circ} 6$, en su resolución 45/111, (14 de diciembre de 1990), disponible en http://www.ohchr.org/SP/Professionallnterest/Pages/

\section{BasicPrinciplesTreatmentOfPrisoners.aspx}

Vidal, T\& Ruiz, M. (2014). Arte, cultura y cárcel. Prácticas artísticas y culturales en contextos penitenciarios. 2017, de Cultura Sin Mesura Sitio web: https://issuu.com/ culturasinmesura/docs/arte_cultura_y_carcel 